\title{
Correction to: Novel algebraic criteria on global Mittag-Leffler synchronization for FOINNs with the Caputo derivative and delay
}

\author{
Yuhong Cheng ${ }^{1}$ (D) $\cdot$ Hai Zhang ${ }^{1}$ (D) $\cdot$ Weiwei Zhang ${ }^{1,2}$ (D) $\cdot$ Hongmei Zhang ${ }^{1}$ (D) \\ Published online: 15 December 2021 \\ (c) Korean Society for Informatics and Computational Applied Mathematics 2021
}

\section{Correction to: Journal of Applied Mathematics and Computing https://doi.org/10.1007/s12190-021-01672-0}

Author discovered proofing errors related with several equations overlooked by typesetter and have been now corrected.

Original article has been updated.

Publisher's Note Springer Nature remains neutral with regard to jurisdictional claims in published maps and institutional affiliations.

The original article can be found online at https://doi.org/10.1007/s12190-021-01672-0.

\section{Hai Zhang}

zhanghai0121@163.com

Yuhong Cheng

chengyuhong26@163.com

Weiwei Zhang

wwzhahu@aliyun.com

Hongmei Zhang

levensky88@163.com

1 School of Mathematics and Physics, Anqing Normal University, Anqing 246133, People's Republic of China

2 College of Science, Nanjing University of Aeronautics and Astronautics, Nanjing 211106, People's Republic of China 